\title{
COMPARACIÓN DEL MÉTODO DIRECTO Y PRECIPITADO, PARA LA DETERMINACIÓN DE LOS NIVELES DE COLESTEROL HDL, EN GALLINAS PONEDORAS
}

\author{
José Henry Osorio ${ }^{1}$ \\ Jancy Darly Flórez ${ }^{2}$
}

Recibido el 3 de diciembre de 2012 y aprobado el 18 de julio de 2013

\section{RESUMEN}

Objetivo: Comparar un método directo y un método de precipitado en la determinación del colesterol HDL en gallinas ponedoras. Metodología: Se obtuvo suero de 40 gallinas ponedoras en ayunas de 26 semanas de edad, de la línea Hy-Line W-36. El colesterol de las lipoproteínas de alta densidad se midió por el método directo a base de un detergente específico junto con N,Nbis(4-sulfobutil)-mtoluidina, y por el método de precipitación a base de fosfotungstato $+\mathrm{Mg}^{2+}$. Resultados: Las medias \pm desviación estándar para los niveles de colesterol de las lipoproteínas de alta densidad, por los métodos directo y precipitado en $\mathrm{mg} / \mathrm{dl}$ fueron de: $63,1 \pm 14,3$; $13,3 \pm 3,5$; respectivamente. El $P$ valor del test $F$ es inferior a 0,05 , evidenciando diferencia estadísticamente significativa, con una confianza del 95\% entre métodos. Conclusiones: Es factible la utilización del método directo a base de un detergente específico y $\mathrm{N}, \mathrm{Nbis}$ (4-sulfobutil)-m-toluidina para la determinación del colesterol de las lipoproteínas de alta densidad en gallinas ponedoras, debido a que es un método que no es alterado por los altos niveles de triglicéridos de estas.

\section{PALABRAS CLAVE}

Detergente + DSBmT, fosfotungstato, lípidos, lipoproteínas.

\section{COMPARISON OF DIRECT AND PRECIPITATION METHODS FOR DETERMINING HDL CHOLESTEROL LEVELS IN LAYING HENS}

\section{ABSTRACT}

Objective: To compare a direct method vs a precipitation method for determining HDL cholesterol in laying hens. Methodology: Serum of forty 26 weeks old fasting laying hens was obtained from the Hy-Line W-36. Cholesterol in high density lipoproteins was measured by direct method based on a specific detergent along with N,Nbis (4sulfobutyl)-m-toluidine, and through the precipitation method based on phosphotungstate $+\mathrm{Mg}^{2+}$. Results: The standard deviation mean \pm for high density lipoproteins though direct and precipitation methods in $\mathrm{mg} / \mathrm{dl}$ were: $63.1 \pm 14.3 ; 13.3 \pm 3.5$ respectively. The $\mathrm{P}$ value for the $F$ test is $<0.05$ showing significant statistical difference between the two analyzed methods with a confidence level of $95 \%$. Conclusions: It is feasible to use the direct method using a specific detergent and $\mathrm{N}, \mathrm{Nbis}$ (4-sulfobutyl)-m-toluidine to determine high 
density lipoprotein cholesterol in laying hens, because this method is not altered by high levels of triglyceride.

\section{KEY WORDS}

Detergente + DSBmT, lipids, lipoproteins, phosphotungstate.

\section{INTRODUCCIÓN}

En las gallinas ponedoras se ha estudiado el metabolismo de los lípidos alterando la dieta, con el fin de aumentar la proporción de los ácidos grasos poliinsaturados en los triglicéridos (TG) y disminuir el colesterol total (CT) de la sangre y de la yema de huevo (Ayerza y Coates, 2000; Chowdhury et al., 2002), el cual finalmente va para consumo humano. Los TG y el CT son transportados en la sangre hasta los oocitos de las gallinas, por las lipoproteínas de muy baja densidad (VLDL, del inglés: very low density lipoprotein) que contienen la apolipoproteína VLDL-II (Walzem et al., 1999) y por las lipoproteínas de alta densidad (HDL, del inglés: high density lipoprotein) que también contienen dicha apolipoproteína (Vieira et al., 1995). En los primeros estudios realizados para separar y cuantificar las lipoproteínas en las aves, se utilizó el método de ultracentrifugación junto con el de electroforesis (Hermier et al., 1984; Hermier et al., 1989) para arrojar resultados del metabolismo de los lípidos en dicha especie. El método de ultracentrifugación, a pesar de la difícil recuperación de las lipoproteínas y a la falta de homogeneidad de las fracciones obtenidas (Palacios et al., 1999), se ha considerado como el método de referencia en combinación con el método de precipitado a base de heparina y cloruro de manganeso, si se requiere medir el colesterol de las HDL (Nauck et al., 2000). No obstante, este método no está totalmente estandarizado en la actualidad, debido a los altos costos operacionales y a la necesidad de personal entrenado para realizar las ultracentrifugaciones. Se han considerado otros métodos más fáciles y asequibles para la cuantificación de las lipoproteínas, pero dichos métodos se basan en la medición del colesterol que transportan las lipoproteínas medidas por ultracentrifugación. Existen diversos métodos para medir el colesterol de las HDL (C$\mathrm{HDL}$ ), entre estos están los métodos de precipitado los cuales consisten en la precipitación de lipoproteínas no HDL, por acción de diferentes reactivos (que varían según el fabricante), y están los métodos directos, igualmente conocidos como homogéneos, los cuales disminuyen costos operacionales comparados con el de ultracentrifugación, estos métodos son específicos y están libres de interferencias endógenas tales como altas concentraciones de TG en sangre (Arranz et al., 1998; Warnick et al., 2001). Recientes investigaciones han estudiado el metabolismo lipídico en aves mediante técnicas de precipitado con fosfotungstato para la cuantificación del C-HDL (Salma et al., 2007; Yin et al., 2008); también han usado el método de ultracentrifugación junto con un método enzimático para los valores del colesterol de dicha lipoproteína (An et al., 1997), y métodos ya sea de precipitado o directos para medir el C-HDL en la sangre de las gallinas ponedoras (Liu et al., 2010; Yue et al., 2011). Sin embargo, en dichos trabajos 
no aclaran la factibilidad de estos métodos, además de mostrar diferencias entre los valores arrojados, los cuales, deberían ya estar estandarizados.

Debido a que los métodos directos se caracterizan porque sus valores no se interfirieren por altos niveles de TG en el suero, en el presente trabajo se comparan los métodos directo [detergente + $\mathrm{N}, \mathrm{Nbis}$ (4-sulfobutílicos)-m-toluidina (DSBmT)] y precipitado (fosfotungstato $+\mathrm{Mg}^{+2}$ ) para la cuantificación del C-HDL, y así dilucidar si la ventaja que posee el método directo, la tiene el método de precipitado.

\section{MATERIALES Y MÉTODOS}

Animales y dieta. Ponedoras de la línea Hy-Line W-36, fueron levantadas en la Granja Montelindo, propiedad de la Universidad de Caldas (Manizales, Colombia), a una temperatura promedio de $25^{\circ} \mathrm{C}$, y 13 horas de luz aproximadamente; fueron alimentadas hasta la semana 24 de edad con una dieta comercial para gallinas ponedoras, junto con todo el lote de aves. Se eligieron completamente al azar las aves a estudiar, manteniéndose en el mismo galpón, y durante las semanas 25 y 26 de edad, se alimentaron con una dieta a base de maíz, torta de soya y aceite de soya (Tabla 1), debido a que no fue posible obtener la información de la composición real de la dieta comercial. La producción durante las semanas 25 y 26 de las gallinas escogidas fue del 96\% (sin tener en cuenta la mortalidad del levante y el alojamiento), con un peso promedio de $1.444 \mathrm{~g}$. Al momento de la toma de las muestras, se realizó un ayuno previo de $13 \mathrm{~h} \pm 1 \mathrm{~h}$. El número de aves fue de 40 , se tomaron $5 \mathrm{~cm}^{3}$ de sangre directamente de la yugular. La sangre se centrifugó a 3000 rpm durante 15 minutos y el suero se congeló a $-30^{\circ} \mathrm{C}$.

Métodos de análisis. Los reactivos para las determinaciones de CT, TG y colesterol HDL pertenecían a los laboratorios Biosystems S.A. (Barcelona, España). Dichos análisis fueron determinados por métodos enzimáticos-colorimétricos, de acuerdo a las recomendaciones del fabricante. Los análisis fueron realizados en un equipo RAYTO RT-1904C, analizador semiautomático de química.

Determinación del colesterol HDL mediante el método de precipitado (fosfotungstato). Para realizar este método se usó 1 $\mathrm{ml}$ de reactivo (fosfotungstato $0,4 \mathrm{mmol} / \mathrm{L}$ y cloruro de magnesio 20 $\mathrm{mmol} / \mathrm{L}$ ), que se mezcló con $0,2 \mathrm{ml}$ de la muestra de suero, se agitó bien y se dejó durante 10 minutos a temperatura ambiente, se centrifugó por 10 minutos a $4000 \mathrm{rpm}$. En el precipitado quedaron las VLDL, IDL y LDL, y en el sobrenadante quedaron las HDL.

Finalmente, con máximo cuidado fueron transferidos $100 \mu \mathrm{l}$ del sobrenadante, depositándolo en otro tubo de ensayo, se mezcló con $1 \mathrm{ml}$ del reactivo para CT (descrito más adelante) y se incubó por 10 minutos en baño maría a $37^{\circ} \mathrm{C}$. El C-HDL fue hidrolizado por la colesterol esterasa y la colesterol oxidasa, esto dio lugar a la 
formación de peróxido de hidrógeno que fue consumido por una peroxidasa en presencia de la 4-aminoantipirina (4-AA) y fenol, quedando como producto final la quinonaimina, siendo este producto proporcional al C-HDL de la muestra, y este se cuantificó espectrofotométricamente.

Determinación del colesterol HDL mediante el método directo (detergente). Este método consta de: un Reactivo $\mathrm{A}$, que contiene un Buffer good, colesterol oxidasa $<1 \mathrm{U} / \mathrm{ml}$, peroxidasa $<1 \mathrm{U} / \mathrm{ml}$, $\mathrm{N}, \mathrm{Nbis}$ (4-sulfobutil)-m-toluidina $1 \mathrm{mmol} / \mathrm{L}$, un acelerador $1 \mathrm{mmol} / \mathrm{L}$. Un Reactivo B, que contiene: Buffer good, colesterol esterasa $<1,5$ $\mathrm{U} / \mathrm{ml}$, 4-AA $1 \mathrm{mmol} / \mathrm{L}$, ascorbato oxidasa <3,0 KU/L y un detergente.

Se tomaron $7 \mu \mathrm{l}$ de la muestra de suero y se le adicionaron $750 \mu \mathrm{l}$ del Reactivo A. Aquí es hidrolizado el colesterol de los portomicrones PM (quilomicrones en mamíferos), VLDL, lipoproteínas de densidad intermedia (IDL, del inglés: intermediate density lipoprotein) y lipoproteínas de baja densidad (LDL, del inglés: low density lipoprotein) por la colesterol oxidasa (CO), en una reacción no formadora de color. Inmediatamente después, se añadieron $250 \mu \mathrm{l}$ del Reactivo B al producto del paso anterior. La mezcla se incubó por 5 minutos a $37^{\circ} \mathrm{C}$. El detergente presente en dicho reactivo solubilizó el colesterol HDL, la colesterol esterasa y la colesterol oxidasa hidrolizaron el colesterol HDL, y dieron como productos finales colestenona y peróxido de hidrógeno; el peróxido de hidrógeno, junto con el DSBmT y la 4-AA, reaccionaron en presencia de una peroxidasa generando o produciendo quinonaimina y cuatro moléculas de agua. La quinonaimina es proporcional a la concentración de colesterol-HDL presente en la muestra, la cual se cuantificó espectrofotométricamente.

Determinación colesterol total (CT). Para la determinación del CT en el suero, se mezclaron $10 \mu \mathrm{l}$ de la muestra y $1 \mathrm{ml}$ de reactivo (PIPES $35 \mathrm{mmol} / \mathrm{L}$, colato sódico $0,5 \mathrm{mmol} / \mathrm{L}$, fenol $28 \mathrm{mmol} / \mathrm{L}$, colesterol esterasa $>0,2 \mathrm{U} / \mathrm{ml}$, colesterol oxidasa $>0,1 \mathrm{U} / \mathrm{ml}$, peroxidasa $>0,8 \mathrm{U} / \mathrm{ml}, 4-\mathrm{AA} 0,5 \mathrm{mmol} / \mathrm{L}, \mathrm{pH} 7,0$ ). Se agitó bien la mezcla y se dejaron incubar los tubos durante 10 minutos, a temperatura ambiente. Los ésteres de colesterol se hidrolizaron por la colesterol esterasa, dieron lugar a colesterol libre, el cual por acción de la colesterol oxidasa formó colestenona + peróxido de hidrógeno, este último en presencia de la 4-AA y fenol dieron lugar a la quinonaimina por acción de la peroxidasa. La quinonaimina es proporcional al colesterol total de la muestra y se cuantificó espectrofotométricamente.

Determinación de triglicéridos (TG). Se utilizaron $10 \mu \mathrm{l}$ de la muestra y $1 \mathrm{ml}$ de reactivo (PIPES $45 \mathrm{mmol} / \mathrm{L}$, 4-clorofenol 6 $\mathrm{mmol} / \mathrm{L}$, cloruro magnésico $5 \mathrm{mmol} / \mathrm{L}$, lipasa $>100 \mathrm{U} / \mathrm{ml}$, glicerolquinasa $>1,5 \mathrm{U} / \mathrm{ml}$, glicerol-3P-oxidasa $>4 \mathrm{U} / \mathrm{ml}$, peroxidasa $>0,8$ $\mathrm{U} / \mathrm{ml}, 4-A A \quad 0,75 \mathrm{mmol} / \mathrm{L}$, ATP 0,9 mmol/L, pH 7,0). Se agitó bien la mezcla y se dejaron dejaron incubar los tubos durante 15 minutos a temperatura ambiente. En el anterior proceso, los triglicéridos fueron hidrolizados por la lipasa a glicerol y ácidos grasos, el glicerol, en presencia de ATP fue fosforilado por la glicerol-quinasa y dio lugar al glicerol 3P + ADP, el glicerol 3P en presencia de oxígeno formó peróxido de hidrógeno por acción de la glicerol-3P-oxidasa. 
Finalmente, se cuantificó espectrofotométricamente la quinonaimina producto de la acción de la peroxidasa sobre el peróxido de hidrógeno en presencia de 4-AA y clorofenol, la quinonaimina es proporcional a la concentración de los TG.

Análisis estadístico. Los datos fueron analizados usando ANOVA simple. Para el análisis estadístico se utilizó Statgraphics Plus 5.1. Las diferencias estadísticamente significativas se determinaron con el $P<0,05$ del test $F$. Se realizó el coeficiente de relación de Pearson para las variables de CT y $\mathrm{CHDL}$ (método directo y precipitado), con una significancia de $\mathrm{P}<0,01$.

\section{RESULTADOS Y DISCUSIÓN}

Cuando se realiza un estudio del metabolismo lipídico en las diferentes especies animales, hay que tener en cuenta que los métodos de evaluación del colesterol de las lipoproteínas son analizados o utilizados en humanos sanos, generalmente ayunados (Arranz et al., 1998), o con enfermedades metabólicas, tales como hipertrigliceridemia (Okazaki et al., 1997) o diabetes (Jensen et al., 2002; Guerra et al., 2005), entre otras. En el caso de las gallinas ponedoras, se han realizado diferentes estudios del metabolismo lipídico con la metodología disponible, mostrando valores específicamente del C-HDL y su baja o alta relación con la yema de huevo, sin tener en cuenta si los datos arrojados corresponden a la realidad de dicho animal, pues los altos niveles sanguíneos de TG en las gallinas pueden afectar los resultados de los métodos utilizados.

En el presente trabajo se realizó el análisis del colesterol de las lipoproteínas de alta densidad, se encontró que los niveles séricos varían significativamente entre los dos métodos analizados. Las medias \pm desviación estándar para los niveles de C-HDL, por los métodos directo y precipitado, en $\mathrm{mg} / \mathrm{dl}$, fueron de: $63,1 \pm 14,3$; $13,3 \pm 3,5$; respectivamente. El $P$ valor del test $F$ es inferior a 0,05 , evidenciando diferencia estadísticamente significativa, con una confianza del 95\% entre métodos (Tabla 2).

Al analizar el método de precipitado con ácido fosfotúngstico $+\mathrm{Mg}^{2+}$, se debe tener en cuenta que dicho método consiste en formar complejos entre las apolipoproteínas B (apo B) de las VLDL, IDL y LDL con los poli-aniones y cationes divalentes que contienen los reactivos (Nauck et al., 2000), y así, lograr la posterior sedimentación de dichas lipoproteínas; sin embargo, este procedimiento se altera cuando hay niveles de triglicéridos superiores a $400 \mathrm{mg} / \mathrm{dl}$ en el suero (Palacios et al., 1999), formando complejos que evitan la precipitación completa de las lipoproteínas no HDL dando como resultado concentraciones de C-HDL superiores a las reales (Harris et al., 1997), aunque, en el inserto de los laboratorios Biosystems, dice que no se presentan problemas con niveles de TG $<1000 \mathrm{mg} / \mathrm{dl}$ (dando la posibilidad de utilizar dicho método en gallinas ponedoras). Teniendo en cuenta, que en las gallinas analizadas en el presente estudio se encontraron niveles de TG entre 396 y $1733 \mathrm{mg} / \mathrm{dl}$ con una media de: 881,5 54345,2 
(Tabla 2), existe la posibilidad de que los valores del C-HDL cuantificados por el método de precipitado con fosfotungstato + $\mathrm{Mg}^{2+}$ sean bajos a la realidad de la gallina ponedora. Sin embargo, dicha alteración no se presentó con falsos positivos, sino al contrario, hubo valores inferiores a lo esperado (Tabla 2), probablemente debido a la presencia de otras apolipoproteínas en las lipoproteínas de las gallinas ponedoras, tales como las VLDL-II (Schneider et al., 1990; Vieira et al., 1995), que no están presentes en las lipoproteínas de los humanos (Newsholme y Leech, 1987), especie donde fueron estudiados los diferentes métodos de evaluación del C-HDL. El método directo para la cuantificación de CHDL, además, de reducir costos operacionales, tiene como ventaja, que inhibe la reacción del colesterol de los PM, VLDL, IDL y LDL y mide directamente el C-HDL, sin afectarse por los altos niveles de TG en suero (Warnick et al., 2001), además, las recomendaciones de los laboratorios Biosystems, dicen que se aceptan valores de TG de $3000 \mathrm{mg} / \mathrm{dl}$, mostrando que dicho método es más seguro de utilizar en las gallinas ponedoras. En la Tabla 3, se presentan los datos de cuatro estudios diferentes, de los niveles sanguíneos del C-HDL de gallina ponedora, con su respectivo método de evaluación o laboratorio responsable de los reactivos. Los niveles de C-HDL encontrados en el trabajo donde analizaron los efectos del ácido linoleico conjugado en la dieta, usando un kit comercial (Beijing Biological Technology Co.) (Yin et al., 2008), a base del método de precipitado con fosfotungstato $+\mathrm{Mg}^{2+}$ (comunicación personal), no encontraron relación entre los niveles de C-HDL y el colesterol total de la yema de huevo. Los datos del C-HDL de otro estudio, donde evaluaron el efecto de bacterias del género Rhodobacter como aditivos en la dieta de gallinas ponedoras, usando un kit enzimático (Wako Pure Chemical Industries Ltd.) (Salma et al., 2007), también a base de fosfotungstato, encontraron que hay una relación inversamente proporcional entre los niveles séricos de C-HDL y los niveles de colesterol de la yema de huevo. Estos dos trabajos, están en el rango de los valores arrojados por el método directo a base de detergentes y DSBmT del presente estudio, pero no coinciden con los valores que arrojó el método de precipitado con fosfotungstato $+\mathrm{Mg}^{2+}$. Por lo tanto, contradicen los valores que se mostraron en la presente investigación, sin embargo, esto puede deberse a que los niveles de TG en dichas investigaciones son aproximadamente de $425 \mathrm{mg} / \mathrm{dl}$ y aparentemente, no habría un efecto negativo en los resultados por dicho método. Los valores del estudio donde usaron varios subproductos del cártamo en la dieta y cebo de ganado vacuno como control, fueron analizados por el método de ultracentrifugación junto con un kit colorimétrico para colesterol (An et al., 1997), los autores mostraron valores por debajo del rango del método directo a base de detergentes y DSBmT, pero, por encima del rango del método de precipitado con fosfotungstato del presente trabajo (Tablas 2 y 3). Particularmente, en dicho trabajo donde utilizaron el método de ultracentrifugación, no encontraron diferencias en los niveles séricos de C-HDL ni en los niveles de colesterol total de la yema de huevo con los cambios que realizaron en la dieta, por lo tanto no muestran si hay correlación entre estos parámetros.

Por otra parte, una investigación donde analizaron el efecto del uso de aceites oxidados en la dieta, sobre el metabolismo de los lípidos, cuantificaron el C-HDL usando un kit enzimático (Beijing BHKT 
Clinical Reagent Co. Ltd.) (Yue et al., 2011). En contraste con los trabajos ya analizados, se observó que los valores se encuentran en el rango de los datos que arrojó el presente estudio por el método de precipitado con fosfotungstato $+\mathrm{Mg}^{2+}$. Conjuntamente, con el trabajo realizado por Liu et al. (2010), donde adicionaron a la dieta esteroles vegetales, tampoco encontraron diferencias significativas en los niveles C-HDL ni en el colesterol de la yema de huevo, con el cambio de la dieta. Aunque varios de los factores que pueden afectar los niveles de C-HDL en la sangre de las gallinas, son el tipo de línea de gallina ponedora (debido a la variabilidad genética) o algunos tipos de dieta utilizados, se pudo dilucidar, que el método de evaluación afecta en gran manera los resultados, por lo tanto, cuando se relacionan con otras variables, los análisis pueden llegar a ser equivocados. No obstante, aunque en el presente trabajo no se midió el colesterol de la yema, sí se cuantificó el CT del suero de las gallinas, encontrándose una media \pm desviación estándar de: $147,4 \pm 37,9$ (Tabla 2).

Al realizar la correlación del CT con el C-HDL evaluado por el método de precipitado con fosfotungstato $+\mathrm{Mg}^{2+}$, no se encontró una correlación entre estas dos variables, pero al realizar la correlación entre el CT y el C-HDL por el método directo a base de detergente + DSBmT, sí se encontró una correlación positiva y significativa entre estas dos variables (Tabla 4), Debido a que las HDLs son unas de las lipoproteínas más importantes en el transporte del colesterol ya sea hacia el hígado o hacía la yema de huevo (Vieira et al., 1995), un método adecuado de evaluación de los niveles de C-HDL podría mostrar una relación o la falta de esta, entre el C-HDL y el colesterol de la yema de huevo.

Tabla 1. Composición de la dieta para gallinas ponedoras $(\mathrm{kg} / 100 \mathrm{~kg})$

\begin{tabular}{lc}
\hline Ingredientes & $\mathrm{kg}$ \\
\hline Maíz & 59,12 \\
Salvado de trigo & 2,65 \\
Torta de soya (48) & 25,72 \\
DL-metionina & 0,22 \\
L-treonina & 0,06 \\
Carbonato de calcio & 8,63 \\
Dicalfos (fosfato bical) & 1,82 \\
Aceite de soya & 1,25 \\
Colina & 0,20 \\
Premezcla de vitaminas y minerales & 0,10 \\
Sal & 0,25 \\
Composición calculada: & \\
Proteína cruda \% & 16,61 \\
Energía metabolizable kcal/kg & 2748,97 \\
\hline
\end{tabular}


Tabla 2. Concentración de colesterol HDL $(\mathrm{mg} / \mathrm{dl})$ por el método directo (detergente y $\mathrm{DSBmT}$ ) y por el método de precipitado (fosfotungstato)

\begin{tabular}{lrc}
\hline \multicolumn{1}{c}{ PARAMETROS } & $\begin{array}{c}\text { C-HDL } \\
\text { Directo }\end{array}$ & $\begin{array}{c}\text { C-HDL } \\
\text { Precipitado }\end{array}$ \\
\hline Media & 63,1 & 13,2 \\
Desviación estándar & 14,3 & 3,5 \\
Mínimo & 38,7 & 6,8 \\
Máximo & 90,9 & 21,3 \\
Rango & 52,2 & 14,4 \\
P-valor & \multicolumn{2}{c}{0,000} \\
\hline C-HDL: Colesterol de la lipoproteína de alta densidad.
\end{tabular}

Tabla 3. Valores aproximados de colesterol HDL $(\mathrm{mg} / \mathrm{dl})$ de gallinas ponedoras, descrito por diferentes autores

\begin{tabular}{lcc}
\hline \multicolumn{1}{c}{ Método } & C-HDL (mg/dl) & Referencia \\
\hline $\begin{array}{l}\text { Ultracentrifugación } \\
+ \text { kit enzimático }\end{array}$ & 37,3 & An et al. (1997) \\
Fosfotungstato & 42,2 & Salma et al. (2007) \\
Fosfotungstato & 54,3 & Yin et al. (2008) \\
$\begin{array}{l}\text { Kit enzimático } \\
\text { Laboratorios: Beijing BHKT Clinical Reagent Co. Ltd. }\end{array}$ & 18,2 & Yue et al. (2011) \\
\hline C-HDL: Colesterol de la lipoprotein a de alta densidad. & &
\end{tabular}

Tabla 4. Coeficiente de correlación de Pearson para las variables de CT, C-HDL método directo y C-HDL método de precipitado

\begin{tabular}{|c|c|c|}
\hline & $\begin{array}{c}\text { C-HDL } \\
\text { Directo } \\
\text { Detergente + DSBmT }\end{array}$ & $\begin{array}{c}\text { C-HDL } \\
\text { Precipitado } \\
\text { Fosfotungstato }+\mathrm{Mg}^{2+}\end{array}$ \\
\hline $\mathrm{CT}$ & $0,6^{\star}$ & 0,04 \\
\hline
\end{tabular}

\section{CONCLUSIONES}

Para la cuantificación de C-HDL en las gallinas ponedoras, es viable utilizar el método directo a base de detergente + DSBmT, debido que este método es adecuado para muestras de suero con niveles de triglicéridos elevados (hasta $3000 \mathrm{mg} / \mathrm{dl}$ ). En el presente trabajo, el método de precipitado con fosfotungstato $+\mathrm{Mg}^{2+}$ mostró que dicho método se afectó negativamente, debido a que arrojó valores significativamente inferiores del colesterol de dichas lipoproteínas, probablemente debido a la presencia de las apilipoproteínas VLDL-II en las HDL de las gallinas ponedoras. 


\section{REFERENCIAS}

- An, B.K., Nishiyama, H., Tanaka, K., Ohtani, S., Iwata, T., Tsutsumi, k. y Kasai, M. (1997). Dietary safflower phospholipid reduces liver lipids in laying hens. Poultry Science, 76(5), 689-95.

- Arranz, M.L., Tasende, J. y Martin, F. (1998). Comparison of two homogeneous assays with a precipitation method and an ultracentrifugation method for the measurement of HDL-cholesterol. Clinical Chemestry, 44(12), 2499-505.

- Ayerza, R. y Coates, W. (2000). Dietary levels of chia: influence on yolk cholesterol, lipid content and fatty acid composition for two strains of hens. Poultry Science, 79(5), 724-39.

- Chowdhury, S.R., Chowdhury, S.D. y Smith, T.K. (2002). Effects of dietary garlic on cholesterol metabolism in laying hens. Poultry Science, 81(12), 1856-62.

- Guerra, M., Luján, D., Alvarado, M., Moreno, D. y Silva, M. (2005). Estudio del perfil lipídico en sujetos con Diabetes Mellitus Tipo 2 de Bogotá. Universitas Scientiarum, 10, 81-89.

- Harris, N., Galpchian, V., Thomas, J., Lannotti, E., Law, T. y Rifai, N. (1997). Three generations of high-density lipoprotein cholesterol assays compared with ultracentrifugation/dextran sulfate-Mg21 method. Clinical Chemistry, 43(5), 816-23.

- Hermier, D., Chapman, J. y Leclercq, B. (1984). Plasma lipoprotein in fasted and refed chickens of two strains selected for high or low adiposity. The Journal of Nutrition, 114(6), 1112-21.

- Hermier, D., Forgez, P., Williams, J. y Chapman, M.J. (1989). Alterations in plasma lipoproteins and apolipoproteins associated with estrogen-induced hyperlipidemia in the laying hen. European Journal of Biochemistry, 184(1), 109-18.

- Jensen, T., Truong, Q., Frandsen, M., Dinesen, B. y Stender, S. (2002). Comparison of a homogeneous assay with a precipitation method for the measurement of HDL cholesterol in diabetic patients. Diabetes Care, 25(11), 1914-8.

- Liu, X., Zhao, H.L., Thiessen, S., House, J.D. y Jones, P.J.H. (2010). Effect of plant sterol-enriched diets on plasma and egg yolk cholesterol concentrations and cholesterol metabolism in laying hens. Poultry Science, 89(2), 270-5.

- Nauck, M., Wiebe, D. y Warnick, G.R. (2000). Measurement of highdensity-lipoprotein cholesterol. In Rifai N., Warnick G.R. y Dominiczak M.H. (Eds.), Handbook of Lipoprotein Testing (pp. 22144). 2nd ed. Washington, D.C.: AACC Press.

- Newsholme, E.A. y Leech, A.R. (1987). Bioquímica Médica. Primera edición. Madrid, España: Interamericana McGraw-Hill.

- Okazaki, M., Sasamoto, K., Muramatsu, T. y Hosaki, S. (1997). Evaluation of precipitation and direct methods for HDL-cholesterol assay by HPLC. Clinical Chemistry, 43(10), 1850-90.

- Palacios, M., Esteban, M., Aguilar, J. y Ortolá, J. (1999). Recomendaciones para la determinación de la concentración en suero de colesterol de las lipoproteínas de alta densidad. Química Clínica, 18(1), 33-40.

- Salma, U., Miah, A.G., Tareq, K.M.A., Maki, T. y Tsujii, H. (2007). Effect of dietary Rhodobactercapsulatuson egg-Yolk cholesterol and laying hen performance. Poultry Science, 86(4), 714-9.

- Schneider, W.J., Carroll, R., Severson, D.L. y Nimpft, J. (1990). Apolipoprotein VLDL-II inhibits lipolysis of triglyceride-rich lipoproteins in the laying hen. Journal of Lipid Research, 31, 507-13. 
- Vieira, P.M., Vieira, A.V., Sanders, E.J., Steyrer, E., Nimpf, J. y Schneider, W.J. (1995). Chicken yolk contains bona fide high density lipoprotein particles. Journal of Lipid Research, 36, 601-10.

- Walzem, R.L., Hansen, R.J., Williams, D.L. y Hamilton, R.L. (1999). Estrogen induction of VLDLy Assembly in Egg-Laying hens. The Journal of Nutrition, 129(2), 467S-72S.

- Warnick, G.R., Nauck, M. y Rifai, N. (2001). Evolution of methods for measurement of HDL-cholesterol: from ultracentrifugation to homogeneous assays. Clinical Chemistry, 47(9), 1579-96.

- Yin, J.D., Shang, X.G., Li, D.F., Wang, F.L., Guan, Y.F. y Wang, Z.Y. (2008). Effects of dietary conjugated linoleic acid on the fatty acid profile and cholesterol content of egg yolks from different breeds of layers. Poultry Science, 87(2), 284-90.

- Yue, H.Y., Wang, J., Qi, X.L., Ji, F., Liu, M.F., Wu, S.G., Zhang, H.J. y Qi, G.H. H. (2011). Effects of dietary oxidized oil on laying performance, lipid metabolism, and apolipoprotein gene expression in laying hens. Poultry Science, 90(8), 1728-36.

1. Laboratorio de Investigación en Bioquímica Clínica y Patología Molecular, Departamento de Ciencias Básicas de la Salud, Universidad de Caldas. Manizales, Colombia. jose.osorio_o@ucaldas.edu.co

2. Facultad de Ciencias Agropecuarias, Universidad de Caldas. Manizales, Colombia.

Para citar este artículo: Osorio, J. H. \& Flórez, J. D. (2014). Comparación del método directo y precipitado, para la determinación de los niveles de colesterol HDL, en gallinas ponedoras. Revista Luna Azul, 38, 122-131. Recuperado de http://lunazul.ucaldas.edu.co/index.php?option=content\&task=v iew\&id $=897$ 